\title{
Comprensión lectora en estudiantes de primero a tercero de Escuela Nueva
}

\section{Reading comprehension in students from first to third grade with "Escuela Nueva" methodology}

Fecha de envío: 26 de noviembre de 2016

Fecha de aceptación: 8 de agosto de 2017

Hugo Alfonso Ávila Cepeda* Rosalbina Jiménez Pinzón **

Jaime Andrés Torres Ortiz ***

Artículo de reflexión

\section{Resumen}

La Ley General de Educación (1994) establece entre los objetivos de Básica, el desarrollo de habilidades comunicativas para leer y comprender. Analizados los resultados de las Pruebas Saber lenguaje $3^{\circ}$ (2014), se observaron bajos desempeños de comprensión lectora en estudiantes de la sede San Pablo con metodología Escuela Nueva (EN). Por esta razón, se implementó una acción didáctica que complementó las guías de EN para el mejoramiento de habilidades lectoras en estudiantes de primero a tercero de primaria, tomando aportes de autores como Henao, Coronel, Thorne, Solé, Jolibert, Blythe, entre otros. Para tal fin, se siguió la metodología de investigación acción cualitativa mediante pruebas diagnósticas, comparativo de resultados entre las anteriores pruebas e implementación de la acción didáctica, con la cual se obtuvo mejor desempeño lector en los estudiantes mediante la realización de
*Institución Educativa Técnica Antonio Nariño Boyacá, Colombia Hugoavila69@yahoo.es **Institución Educativa Técnica Antonio Nariño Boyacá, Colombia rosaljim77@gmail.com Universidad Pedagógica y

***Tecnológica de Colombia - Boyacá, Colombia Jaime.torres@uptc.edu.co

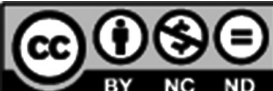


varias actividades en torno a la lectura, viéndose involucrada la transformación de la práctica pedagógica del docente.

\section{Abstract}

The General Education Law (1994) establishes among the objectives of elementary school, the development of communicative skills to read and understand. Analyzed the results of the "SABER" tests for third grade in language (2014), low reading comprehension performances were observed in students of the San Pablo campus with Escuela Nueva (EN) methodology. For this reason, a didactic action was implemented that complemented the EN guidelines for the improvement of reading skills in students from first to third grade of primary school, taking contributions from authors such as Henao, Coronel,
Palabras clave: Comprensión lectora, Escuela Nueva, Acción didáctica, investigación acción, Pruebas saber.

Thorne, Solé, Jolibert, Blythe, among others. For this purpose, the methodology of qualitative researchaction was followed through diagnostic tests, comparative results between the previous tests and implementation of the didactic action, with which better reading performance was obtained in the students by carrying out several activities around reading, being seen the transformation of the pedagogical practice of the teacher.

Keywords: reading comprehension, New School, didactic action, action research, SABER Tests. 


\section{Introducción}

La Comprensión Lectora es la capacidad que desarrolla el ser humano para abstraer el mensaje contenido en un texto, y referirse a la base fundamental del significado de la información presentada. Blythe (2002) afirma: "que el acto de comprender es poder llevar a cabo una diversidad de acciones $\mathrm{O}$ desempeños que demuestren que la persona entiende el tópico y al mismo tiempo lo amplía” (p. 40). Por tanto, cuando el estudiante comprende lo que lee, es porque tiene la habilidad para entrelazar los conocimientos que posee con la nueva información, dándole un valor útil para su formación personal.

Dada la importancia de la Comprensión Lectora en el desarrollo del aprendizaje, se encuentran ideas concordantes entre diferentes teóricos y los lineamientos establecidos por el Ministerio de Educación Nacional (MEN), constituyéndose la competencia comunicativa lectora, la base fundamental para el crecimiento intelectual del estudiante. Se establece como uno delos objetivos dela educación básica, "desarrollar las habilidades comunicativas para leer, comprender, escribir, escuchar, hablar y expresarse correctamente" (Ley 115, 1994, p. 30) para interactuar espontáneamente en la sociedad mediante el aporte de ideas transformadoras.

Sin embargo, las investigaciones realizadas a nivel nacionaleinternacional, reportan que los estudiantes presentan bajo nivel de comprensión lectora, situación que no es ajena a la Institución
Educativa Técnica Antonio Nariño del Municipio de San Eduardo, ya que dicha problemática es evidente en los estudiantes de grado tercero de educación básica primaria, reflejada en los resultados de las pruebas SABER 2014, con un desempeño débil en el componente sintáctico y muy débil en el componente semántico de la competencia comunicativa lectora.

De igual manera, esta situación problema fue observada en el aula de clase de la Sede San Pablo de la Institución Educativa en mención, donde el aprendizaje es orientado con metodología EN. En dicha sede, los estudiantes presentaron dificultad para desarrollar actividades pedagógicas planteadas en las cartillas de aprendizaje y las diseñadas por la docente, ya que leen despacio por sílabas, cambian palabras, repiten varias veces la misma sílaba, se distraen y tienen dificultades para argumentar y seguir instrucciones.

Considerando lo anterior, se plantea la pregunta: ¿Qué acciones didácticas pueden complementar las guías de aprendizaje de EN para mejorar la comprensión lectora en los estudiantes de grados primero a tercero de Educación Básica Primaria de la sede San Pablo? Con base en la metodología de investigación acción, se analiza la inmersión del docente en su realidad inmediata entendida como el contexto de aula (Elliott, 2002).

Así mismo, el proceso investigativo se realiza en tres fases: la fase diagnóstica, que consta del desarrollo de una Bitácora con actividades propias del material EN y un ejemplo de prueba
La Comprensión Lectora es la capacidad que desarrolla el ser humano para abstraer el mensaje contenido en un texto, y referirse a la base fundamental del significado de la información presentada 


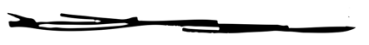

En la metodología de EN, se identifica en el estudiante el aprendizaje activo, participativo y colaborativo. Además, favorece la observación y exploración mediante el contacto directo con el objeto en estudio, despertando la curiosidad y creatividad (Colbert, 2009).
SABER diseñada por el ICFES, dando paso al primer objetivo que consiste en diagnosticar el desempeño en la competencia comunicativa lectora de los estudiantes de grado tercero de educación básica primaria de Escuela Nueva.

En la fase comparativa, se realiza un paralelo entre las pruebas anteriores, dando cumplimiento al segundo objetivo que es comparar con base en el diagnóstico de comprensión lectora, las competencias básicas obtenidas en las guías de aprendizaje, con los componentes establecidos en las pruebas SABER. Por último, el desarrollo del tercer objetivo, diseñar e implementar la acción didáctica, que complementa las guías de aprendizaje Escuela Nueva y fortalece la comprensión lectora en los estudiantes de los grados primero a tercero de primaria.

Con la aplicación de la Acción Didáctica, se fomentó las relaciones interpersonales adecuadas entre estudiante estudiante y estudiantes docente, el trabajo colaborativo y de ayuda entre los estudiantes de diferente grado, la participación activa, dinámica, de diálogo y de intercambio de ideas, que facilitó a los estudiantes la comprensión de los textos narrativos leídos, logrando mejor desempeño en cada uno de los componentes de la competencia comunicativa lectora.

\section{Antecedentes}

El recorrido investigativo y conceptual, ha permitido identificar ideas concernientes a la comprensión lectora en estudiantes de educación básica primaria, haciendo una aporte al conocimiento sobre la realidad objeto de estudio, en donde se clasificaron las temáticas de la siguiente forma: Estrategias Lúdico didácticas, pruebas de lectura de textos, pruebas de pre test y pos test, y uso de Tecnologías de Información y Comunicación (TIC).

Teniendo en cuenta la clasificación, se encuentran aportes investigativos que analizan el juego lúdico para que los estudiantes de E.N. logren relacionar y decodificar el lenguaje (Coronel, 2015). Puesto que, cuando se diseña una acción didáctica con un propósito bien definido, se logra obtener mejores niveles de comprensión, sobre todo lo relacionado con el juego, ya que esta experiencia es agradable a la edad de los estudiantes de primero a tercero y fortalece al aprendizaje significativo.

En la metodología de EN, se identifica en el estudiante el aprendizaje activo, participativo y colaborativo. Además, favorece la observación y exploración mediante el contacto directo con el objeto en estudio, despertando la curiosidad y creatividad (Colbert, 2009). De esta forma, el conocimiento es adquirido a través de los diferentes espacios que hay en la escuela, donde se encuentran elementos que facilitan experimentar, armar, transformar y sacar conclusiones que permitan fortalecer el aprendizaje a partir de diversas experiencias.

Con respecto a la temática de Prueba de lectura de textos, se halla el uso de la evaluación como instrumento para el reconocimiento de la información explícita y la organización del texto 
en términos de su coherencia y cohesión, para luego determinar las estrategias pedagógicas o experiencias de aprendizaje que mejoren el proceso (Henao, 2011). Lo anterior; es motivo de reflexión, ya que no basta con emitir juicios sobre la baja comprensión lectora, sin antes haber hecho un trabajo investigativo, mediante la aplicación de instrumentos, y tampoco basta con hacer un diagnóstico; sino que, con base en los resultados, diseñar y aplicar una acción didáctica que estimule la lectura comprensiva. "Por ende, es fundamental comprender que la reflexión y prácticas propias del docente deben estar encaminadas a reconocer su experiencia, el saber que se produce de ellas y a visibilizar las dificultades y oportunidades caracterizadas en los distintos espacios de formación." (Cañizalez \& Benavides, 2016, p. 113)

De las investigaciones que refieren al uso de Pruebas de Pre- test y Postest, se encuentran las estrategias de la Secuencia Didáctica para comprender textos narrativos, realizada en varias fases: un pre-test de diagnóstico, una secuencia didáctica con enfoque interactivo, un pos-test evaluativo y la comparación de los resultados del pretest y pos-test. Estos momentos de evaluación evidenciaron una mejoría continua en los estudiantes de grado tercero en cuanto a la comprensión lectora (Castañeda et al., 2015). Lo anterior; refleja que, al ser implementado el enfoque interactivo; en el desarrollo de actividades planteadas en las guías de aprendizaje EN, facilita la comprensión de las mismas por parte de los estudiantes.
De la última clasificación, se consideran adecuadas las estrategias de comprensión de lectura mediadas por el uso de la Plataforma Virtual ICON, donde se evidencia alto rendimiento en compresión a través del uso de esta plataforma (Thorne et al., 2012). El empleo de este tipo de herramientas educativas virtuales, se ha convertido en un medio para acceder a la información de manera rápida y divertida, a la vez que mantiene activo el interés de los estudiantes, para explorar sobre los diferentes temas que allí se presentan.

Según lo expuesto en los antecedentes, leer comprensivamente es indispensable porque le permite al lector entrelazar los conocimientos que posee con la nueva información que le suministran los textos, para construir un nuevo significado de lo que pretende conocer. Por tanto, en la sede San Pablo, se hace necesario implementar en el aula estrategias de lectura acordes al grado y edad de los estudiantes, para mejorar el nivel de comprensión lectora que, fortalecida desde los primeros años de escolaridad, desarrolla habilidades comunicativas que contribuyen al aprendizaje y hace posible mejorar los resultados de las pruebas internas y externas e índices sintéticos de calidad de la institución educativa.

Para fundamentar la investigación base del presente artículo, se tomaron varias teorías planteadas, con el propósito de ubicar el problema abordado, dentro de unos conocimientos sólidos que permitan orientar el procesoinvestigativo, argumentar adecuadamente y conocer más el tema de la comprensión lectora.
Según lo expuesto en los antecedentes, leer comprensivamente es indispensable porque le permite al lector entrelazar los conocimientos que posee con la nueva información que le suministran los textos, para construir un nuevo significado de lo que pretende conocer. 
De igual manera, Blythe (citado en Baquero \& Ruiz, 2005) sostiene que: "La comprensión requiere por su parte la capacidad de hacer alrededor de un tema una variedad de cosas que estimulan el pensamiento"
$\mathrm{Al}$ respecto, Solé (1992) manifiesta que: "leer es un proceso de interacción entre un lector y un texto" (p. 100). Es decir, que antes de la lectura, el estudiante posee unos saberes sobre el texto. Al leer, este puede ampliar su conocimiento, tornándose interesante en la medida que responde a sus necesidades e intereses, a la vez que le permite desarrollar la imaginación e interpretación.

De igual manera, Blythe (citado en Baquero \& Ruiz, 2005) sostiene que: "La comprensión requiere por su parte la capacidad de hacer alrededor de un tema una variedad de cosas que estimulan el pensamiento" (p. 76). Por tanto, es indispensable iniciar el proceso de lectura, siguiendo estrategias con actividades variadas acordes a la edad, donde el lector se sienta atraído a conocer el mundo mediante la información suministrada por los libros.

Sin embargo, es importante resaltar que el estudiante adquiere la capacidad para transformar grafemas y símbolos en palabras, oraciones y párrafos, dándole significación a la manera como estén ordenadas, y sintiendo la necesidad de leer más para instruirse. En consonancia con lo anterior, Jolibert (2002) manifiesta que: "Es leyendo que uno se transforma en lector y no aprendiendo primero para poder leer después" (p. 25). Por consiguiente, se requiere la práctica constante de la lectura, para desarrollar habilidades de la competencia comunicativa lectora en sus componentes (semántico, sintáctico y pragmático), facilitando abstraer el mensaje contenido en el texto, cada vez con mayor fluidez y rapidez.
Así mismo, Solé (2011) establece que "la relación entre leer, comprender y aprender constituyen una competencia fundamental en educación primaria que es la competencia de aprender a aprender"'(p. 245). Así pues, el docente debe hacer una reflexión sobre su quehacer pedagógico, siendo creativo en la planeación y aplicación de estrategias que fortalezcan el aprendizaje por medio de la lectura; $y$, una vez terminado el ciclo de Básica Primaria, el estudiante demuestre los saberes adquiridos en los diferentes ámbitos de la vida.

La práctica de la lectura en las escuelas, es un objetivo primordial, por tanto en el aula de clase se debe disponer de textos al alcance y acordes al estudiante, para que realice voluntariamente esta práctica. Por consiguiente, Solé (2011) piensa que: "conseguir que los alumnos aprendan a leer correctamente es uno de los múltiples retos que la escuela debe afrontar puesto que la adquisición de la lectura es imprescindible para moverse con autonomía en las sociedades letradas" (p.27). Además, el docente cumple una función de ayuda cuando facilita este proceso, mediante pautas o estrategias para que el estudiante pueda sacar sus propias conclusiones sobre lo leído.

Teniendo en cuenta la lectura como un proceso amplio de percepción e interpretación de diferentes contextos, es necesario partir de los conocimientos previos. Al respecto, Solé (citado en Barriga \& Hernández, 2010), indica que "comprendemos porque podemos establecer relaciones significativas entre lo que ya sabemos, hemos vivido o 
experimentado y lo que el texto nos aporta" (p. 247). Esto facilita al lector la comprensión de lo que lee, mediante el desarrollo de habilidades para abstraer la intención comunicativa del autor del texto y luego hacer aportes al mismo.

De ahí que, al leer un texto se requiere comprender su contenido. Para lograrlo, Solé (2011) manifiesta que: "en esta comprensión interviene tanto el texto, su forma y su contenido, como el lector, sus expectativas y sus conocimientos previos" (p.18). Por esta razón, en los estudiantes que inician su vida académica, es importante tener en cuenta una secuencia en la práctica de la lectura, partiendo de los saberes que ellos tienen sobre el tema, presentar textos llamativos de acuerdo con su etapa de desarrollo, con contenidos interesantes y con la ayuda de otra persona logre una adecuada comprensión, evitando posibles frustraciones y apatías a la lectura.

Como existe una gran variedad de tipos de textos, cada uno cumple una función específica en el lector, con cierta complejidad para su comprensión; sin embargo, es necesario que los estudiantes los reconozcan. Por consiguiente, Cooper (citado en Solé, 2011) clasifica los textos en "dos tipos básicos de texto, los narrativos y los expositivos" (p. 73). Pero, en los primeros años de escolaridad, es adecuado enfocarlos a que aprendan a leer cierto tipo de texto que sea cercano a las vivencias personales y de fácil comprensión, para luego motivarlos a leer otras clases de textos distintos (Solé, 2011).

Por tal razón, en esta investigación se implementó la acción didáctica a través de los textos narrativos, para el proceso de comprensión lectora en estudiantes de primero a tercero de primaria, ya que dichos textos tiene como función principal divertir $y$, en algunos casos, dejar una enseñanza o moraleja al lector. Básicamente, están conformados por un escenario, personajes (principal y secundario); se narra la secuencia de acciones que se presentan en inicio, nudo y desenlace, lo que hace que el estudiante recuerde los eventos narrados, se usan frases estilísticas como "En un lejano país...", "Érase una vez..." (Barriga y Hernández, 2002). Además, permite al lector, relacionar los hechos narrados con los de su diario vivir.

Además, teniendo en cuenta la edad, y grado de desarrollo intelectual en que se encuentran los estudiantes tomados como muestra en la presente investigación, es importante incluir el juego en las actividades establecidas para la clase, dado que cumple el papel de intercesor de procesos educativos, ya que permite al estudiante, crear lazos de confianza y motivación para involucrarse en las actividades académicas (Duarte, 2003). Promover el juego en el aula de clase, desarrolla en los participantes, habilidades para relacionar y decodificar los textos con mayor facilidad (Coronel, 2015).

Teniendo en cuenta que las guías de aprendizaje son dispositivos interactivos fundamentales del componente curricular del Sistema Escuela Nueva, en este sentido, Mogollón (2011) afirma que: "Las guías ponen en manos de los estudiantes el currículo para que ellos mismos lo administren. Ellos se convierten en
Como existe una gran variedad de tipos de textos, cada uno cumple una función específica en el lector, con cierta complejidad para su comprensión; sin embargo, es necesario que los estudiantes los reconozcan. 


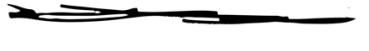

Para reunir información de la muestra en estudio, se sigue la metodología de investigación acción. forjadores activos de un aprendizaje funcional y aplicado a la vida" (p.66). Por tanto, son textos que promueven el aprendizaje autónomo, activo y participativo, centrado en el estudiante, mediante el desarrollo de actividades paso a paso de manera individual, en pareja, o en equipo, para llegar a la respuesta de las preguntas iniciales de cada guía.

Por otro lado, es de considerar que la Acción Didáctica tiene un eje temático contextualizado, que debe coincidir con las preferencias, interesesycaracterísticas psicológicas de los estudiantes, al tiempo debe permitir ordenar coherentemente los componentes curriculares, ello implica un conocimiento claro de los objetivos de enseñanza y aprendizaje por parte del docente (Solé, 2011). Los ejes temáticos deben concretarse con claridad, diferenciando entre los contenidos básicos o fundamentales y aquellos que se proponen para ampliar o profundizar en determinados conocimientos, habilidades o aptitudes.

Por consiguiente, para enseñar a leer, se propone una Acción Didáctica con estrategias de lectura, sobre lo cual Solé, considera relacionarlo a los procedimientos. Por lo tanto, Coll (citado en Solé, 2011) afirma que: "Un procedimiento es como un conjunto de acciones ordenadas y finalizadas, es decir, dirigidas a la consecución de una meta" (p.58). Entonces, es pertinente enseñar a leer; mediante el desarrollo de actividades en cada uno de los momentos de la lectura: antes, durante y después (Solé, 1992). Dicha estrategia, permite el desarrollo de habilidades para comprender y argumentar los propósitos del autor, dándole la posibilidad de adquirir un nuevo aprendizaje para la superación de su intelecto personal y social.

\section{Propuesta metodológica}

Para reunir información de la muestra en estudio, se sigue la metodología de investigación acción. Este método se sustenta en el paradigma cualitativo con enfoque descriptivo, analítico e interpretativo, en donde la inmersión del docente en el aula facilita el conocimiento a través de las interacciones y relaciones mediadas por los sentidos (Elliott, 2002). En estas situaciones, está presente la influencia de la acción didáctica, y el uso de cartillas de aprendizaje para el desarrollo de los procesos académicos en los estudiantes de EN.

Por consiguiente, para este proceso se tuvo en cuenta la fase inicial diagnóstica, fase de comparación de las experiencias y fase de implementación, en las cuales se utilizaron técnicas e instrumentos que permitieron la organización de la información recolectada a través de la observación en el aula y el desarrollo de las guías de aprendizaje, facilitando con ello, el respectivo análisis einterpretación de los datos obtenidos en el proceso, que a la vez permitieron identificar nuevas estrategias para la transformación de la práctica pedagógica, fortaleciendo así, la comprensión lectora en los estudiantes de primero a tercero de primaria.

\section{Alcances}

En primera instancia, se desarrolló la fase inicial diagnóstica, mediante la aplicación de la Bitácora Uno basada en actividades de las guías de EN, y un 
ejemplo de Pruebas SABER Lenguaje $3^{\circ}$ (ICFES, 2012), establecidas para el primer objetivo, que es diagnosticar el desempeño en la competencia comunicativa lectora de los estudiantes de grado tercero de primaria. La información se recogió en el formato de evaluación de competencias lectoras, como medio facilitador para el posterior análisis descriptivo. Además, todas las acciones ocurridas en el aula, son la base para una reflexión inicial y esta da origen a una reflexión posterior o metaanálisis, en donde se determinan los resultados obtenidos.

Mediante la fase comparativa, se hace el desarrollo del segundo objetivo a través de un paralelo entre los resultados obtenidos en la Bitácora Uno con los resultados del ejemplo de prueba saber, con el fin de identificar el nivel de comprensión lectora que poseen los estudiantes tomados como muestra en esta investigación y además establecer si existe coherencia o no entre las competencias desarrolladas en las cartillas de aprendizaje de E.N. y los componentes evaluados por el ICFES. Para facilitar la interpretación de los resultados de manera descriptiva, se empleó la rejilla de relación entre los desempeños de la prueba saber y los obtenidos en las guías de aprendizaje EN (ver tabla 1).

Tabla 1

Rejilla de relación Prueba Saber - Guías de aprendizaje

\begin{tabular}{|l|l|l|l|l|l|l|}
\hline \multicolumn{3}{|c|}{ Prueba Saber } & \multicolumn{3}{c|}{$\begin{array}{c}\text { Guía de Aprendizaje Escuela } \\
\text { Nueva }\end{array}$} \\
\hline \multirow{2}{*}{$\begin{array}{c}\text { Criterios } \\
\text { Componente. }\end{array}$} & Avanzado & Satisfactorio & Mínimo & Excelente & Sobresaliente & Aceptable \\
\hline $\begin{array}{l}\text { Componente } \\
\text { semántico }\end{array}$ & & & & & & \\
\hline $\begin{array}{l}\text { Componente } \\
\text { Sintáctico }\end{array}$ & & & & & & \\
\hline $\begin{array}{l}\text { Componente } \\
\text { Pragmático }\end{array}$ & & & & & & \\
\hline Ryyyyyy \\
\hline
\end{tabular}

Autor, 2015

Teniendo en cuenta los anteriores hallazgos, que sirvieron a la fase comparativa, se procedió al desarrollo del tercer objetivo mediante la fase complementaria, en la cual se diseñó la Acción Didáctica a través del instrumento la Bitácora Dos y Bitácora Tres con actividades establecidas para los tres momentos de lectura (Solé, 2011).
Dichas actividades complementan las guías de $\mathrm{EN}$, en lo referente a los textos narrativos, ya que antes de la lectura se incluye el juego para crear ambientes de aprendizaje, que motivan al estudiante a la participación y se incluyen otras actividades a las planteadas en la guía, para lograr mejor comprensión lectora en los estudiantes (ver Tabla 2). 
Tabla 2

\begin{tabular}{|l|l|}
\hline Momentos de la lectura & Actividades \\
\hline Antes de la lectura. & -La fábula y sus partes. \\
-Dinámica "El teléfono & -¿Qué es un leñador? \\
roto". & -¿Qué hacen los leñadores? \\
-Activación de & -¿Qué herramienta utiliza un leñador? \\
conocimientos previos. & $\begin{array}{l}\text {-Elaborar predicciones o hipótesis, acerca de lo que tratará } \\
\text { el texto y cómo lo dirá. } \\
\text {-Elaboración de preguntas. }\end{array}$ \\
\hline $\begin{array}{l}\text { Durante la lectura. } \\
\text {-Lectura de la fábula "El } \\
\text { Leñador y el espíritu de } \\
\text { las aguas". }\end{array}$ & $\begin{array}{l}\text {-Los estudiantes realizarán pseudo lectura del texto. } \\
\text {-Subrayado de ideas principales y palabras desconocidas. } \\
\text {-Diálogo sobre la lectura. }\end{array}$ \\
\hline $\begin{array}{l}\text { Después de la lectura. } \\
\text {-Análisis de la fábula "El } \\
\text { leñador y el Espíritu de } \\
\text { las aguas". }\end{array}$ & $\begin{array}{l}\text {-Identificar personajes, ambiente y tiempo. } \\
\text {-Relacionar palabras del texto con su significado. } \\
\text {-En grupos de tres estudiantes, ordenar los párrafos } \\
\text { entregados en desorden. } \\
\text {-Ordenar viñetas y texto en forma de friso. } \\
\text {-En grupo, establecer la enseñanza o moraleja que deja la } \\
\text { fábula. }\end{array}$ \\
\hline
\end{tabular}

La intención de este trabajo investigativo es demostrar que al implementar varias estrategias o acciones en la práctica de lectura, se logra fortalecer la comprensión lectora en los estudiantes, despierta el interés y gusto por la práctica, tanto en el aula de clase como fuera de ella, desarrollando su autonomía para seguir sin dificultades en los futuros niveles de formación académica e intelectual.

\section{Resultados obtenidos}

De acuerdo con el desarrollo de la fase diagnóstica, mediante la Bitácora Uno, se refleja que los estudiantes de grado tercero de la sede San Pablo, presentan mayor dificultad para comprender textos que tienen que ver con el para qué se dice, en función de la situación de comunicación (componente pragmático), y determinar la organización del texto en términos de su coherencia y cohesión, es decir, cómo se dice en dicho texto (componente sintáctico), y presentan un menor grado de dificultad en lo relacionado por el qué se dice en el texto (componente semántico).

En la aplicación del ejemplo de Prueba Saber, los resultados indican que los estudiantes de grado tercero, presentan 
mayor dificultadparacomprenderlosítems relacionados al componente pragmático, los ítems para los componentes semántico y sintáctico logran comprenderlos adecuadamente. Al realizar el paralelo entre los anteriores resultados mediante la fase comparativa, se evidenció que los estudiantes de grado tercero de primaria contestaron satisfactoriamente a las preguntas relacionadas al componente semántico.

En cuanto a las preguntas del componente sintáctico, obtuvieron un nivel mínimo; y, en las establecidas para el componente pragmático, quedaron en nivel insuficiente. Además, permitió constatar que efectivamente las competencias que se desarrollan mediante las cartillas de escuela nueva equivalen a los componentes que evalúan las pruebas SABER.

A través de la fase de implementación, se aplica la acción didáctica, teniendo como propósito mejorar los niveles de comprensión lectora en los estudiantes de primero a tercero de primaria, diseñada con estrategias que buscan desarrollar el interés y gusto por la lectura, fijando la atención en el desarrollo de las actividades propuestas, interactuando con los compañeros en los grupos y equipos de trabajo, incrementando la confianza en sí mismo y en el profesor; que, con su actitud frente a este proceso, se convierte en modelo a seguir para el fomento del hábito lector en los estudiantes.

Teniendo en cuenta los hallazgos de la fase comparativa, se diseñó la acción didáctica a través del instrumento la Bitácora dos, con actividades que incluyen el juego considerado como una herramienta pedagógica "vista como un saber que se preocupa por la formación del ser humano en todas sus dimensiones (ser, saber y hacer)" (Orrego \& Toro, 2014, p. 13

3) que dinamiza procesos de aprendizaje siendo de muy buena aceptación en los estudiantes, predisponiéndolos para participar en el desarrollo de las actividades posteriores, de igual manera, se realiza la lectura con actividades previamente definidas para cada uno de los momentos antes, durante y después.

En consecuencia, se desarrollan actividades antes de la lectura, donde se presenta el tema, el objetivo y, a la vez, se indaga sobre los saberes que poseen los estudiantes, dándoles la oportunidad de lanzar hipótesis sobre el tema y manifestar sus puntos de vista con seguridad y claridad, fortaleciendo la expresión oral para expresar las ideas cada vez con más seguridad y libres de temores, habilidad que es muy importante fortalecer desde los primeros años en el estudiante del sector rural, que de acuerdo con su contexto poco tiene oportunidad de expresar sus ideas y puntos de vista con claridad ante un grupo.

Teniendo en cuenta lo anterior, se inicia la lectura de manera alternada en grupo, siguiendo actividades durante la misma, las cuales fortalecen en el estudiante el desarrollo de habilidades lectoras, pues se motivan unos a otros para fijar la atención. Igualmente, cuando la docente hace la lectura, los estudiantes la siguen, lo cual permite corregir la pronunciación, aterrizándolos en la acentuación y modulacióndelas pausas correspondientes a los signos de puntuación, permitiendo mayor comprensión al texto leído.
A través de la fase de implementación, se aplica la acción didáctica, teniendo como propósito mejorar los niveles de comprensión lectora en los estudiantes de primero a tercero de primaria, diseñada con estrategias que buscan desarrollar el interés y gusto por la lectura 


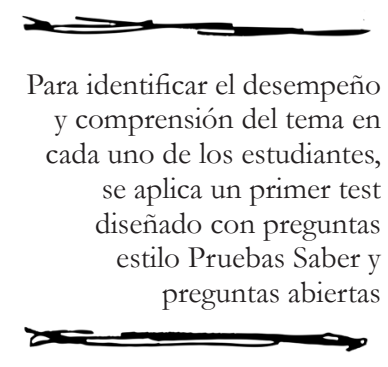

No obstante, en el desarrollo de actividades posteriores a la lectura, se organizan los estudiantes en pequeños equipos, a quienes se le entrega materiales, incluyendo el cuento fraccionado, y se les orienta para identificar la idea principal y cada una de las partes, para luego ordenarlo y reconstruirlo de forma coherente, actividades que invitan a la interacción entre los estudiante para analizar y encontrarle sentido a la misma. Una vez ordenado el texto, socializan el trabajo ante los otros equipos, fortaleciendo habilidades comunicativas de expresión oral.

Para concluir la actividad, siguiendo las orientaciones de la docente, se unen los grupos, entre ellos nombran un líder y distribuyen funciones para reconstruir coherentemente el material impreso por partes, mediante un friso donde se relacionan imágenes y texto. Luego, es expuesto en la cartelera "Mis trabajos", el cual es observado y leído con mucha curiosidad, especialmente por los estudiantes que están comenzando el proceso de lectura, pues al relacionar imágenes con texto descifran el mensaje de manera clara convirtiéndose en una herramienta que les facilita la comprensión del texto, mientras que eleva los niveles de autoestima y se ven motivados a seguir practicando la lectura.

En síntesis, se pudo evidenciar que el diseñar una acción didáctica con actividades variadas alrededor de un tema, favorece y dinamiza la atención e interés de los estudiantes, pues se ven muy motivados en el desarrollo de las actividades, dándose el aprendizaje colaborativo entre los participantes; y, a la vez, se facilita y optimiza el trabajo de la docente que orienta a varios grupos, además se fortalecen las relaciones, se facilita el conocimiento, pues al interactuar al interior del grupo aprenden unos de otros dando paso al aprendizaje significativo.

Para identificar el desempeño y comprensión del tema en cada uno de los estudiantes, se aplica un primer test diseñado con preguntas estilo Pruebas Saber y preguntas abiertas, relacionadas con los componentes semántico, sintáctico y pragmático de la competencia comunicativa lectora, cuyos resultados fueron registrados en el instrumento formato de competencias lectoras, para luego realizar su respectivo análisis descriptivo.

En resumen, se observa que los estudiantes alcanzan un desempeño avanzado en el componente semántico, y satisfactorio en cuanto a los componentes sintáctico y pragmático, viéndose un progreso en los resultados. De esta manera, es evidente que una actividad planeada permite que los estudiantes alcancen los objetivos propuestos. Por esta razón y como complemento a la bitácora Dos, se diseñó la bitácora Tres, por tanto se siguió los mismos momentos, solo se cambió unas actividades, ejemplo: en lugar de friso, se planteó completar crucigrama, en lugar de ordenar párrafos fraccionados, se antepone el número ordinal a cada párrafo que aparece en desorden para darle la secuencia al texto.

En el desarrollo de actividades antes de la lectura, los estudiantes elaboran predicciones, se formulan preguntas acerca de los posibles hechos que suceden 
en el cuento, lo que les causa curiosidad y los mantiene atentos y motivados en el desarrollo de las actividades durante la lectura, donde se observó el gusto y dedicación dándose un alto nivel de comprensión, evidenciado en el desarrollo de las actividades después de la lectura, al identificar la idea principal, características de los personajes, solución de crucigrama relacionando las palabras con su significado y dando respuesta a cuestionario con preguntas tipo saber, donde obtuvieron desempeños del nivel avanzado.

En definitiva, es evidente que del interés y actitud que tenga el docente para indagar sobre estrategias innovadoras acerca de la lectura y que a su vez le permitan diseñar acciones didácticas con objetivos bien definidos, que se reflejen en el alcance de los mismos, conllevan a que el docente reflexione sobre la efectividad de las prácticas en el aula, las cuales se entienden como el espacio donde ocurren procesos de enseñanza y aprendizaje, y a que replantee el quehacer pedagógico para convertirse en puente que acerque, de una manera lúdica, al estudiante hacia la adquisición de nuevos saberes para fortalecer su aprendizaje.

En conclusión, es importante resaltar que el docente de Educación Básica
Primaria es medio de ayuda pedagógica y modelo a seguir para que el estudiante se acerque al conocimiento por medio de la lectura, por tanto, le es un reto indagar para transformar su práctica demostrando buena actitud en el diseño de estrategias adecuadas e innovadoras que despierten en el lector el gusto e interés para explorar el conocimiento contenido en los textos, desarrollando su pensamiento crítico y la capacidad de creación (Samacá, 2016), lo que beneficiara su proceso de formación como ser integral y de éxito.

Considerar al estudiante protagonista de su aprendizaje, es brindarle espacios para interactuar con los compañeros y el docente, en un ambiente de confianza, de diálogo para compartir saberes o experiencias, actuando con espontaneidad y claridad en sus apreciaciones. Con el desarrollo de la Acción Didáctica, los estudiantes se mostraron activos en cada actividad, se reflejó la ayuda entre estudiantes de diferente grado logrando todos la comprensión del texto, es decir, se presentó el trabajo colaborativo, manteniendo las buenas relaciones interpersonales, con oportunidad de analizar, comparar, reflexionar y ampliar su conocimiento constantemente mediante la práctica de la lectura. 


\section{Referencias}

Baquero, P., \& Ruiz, H. (2005). La enseñanza para la comprensión: una visión integradora de los fundamentos y estrategias de la enseñanza. Actualidades Pedagógicas, 1(46), 75-83.

BARRIgA, F., \& HERNÁNDEZ, G. (2010). Leer y escribir para un aprendizaje significativo y reflexivo. En Estrategias docentes para un aprendizaje significativo. Una interpretación constructivista. (pp. 224-299). México: Mc-Graw-Hill.

Barriga, F., \& Hernández, G. (2002). Estrategias docentes para un aprendizaje significativo. Una interpretación constructivista. México: Mc-Graw-Hill.

Blythe, T. (2002). La enseñanza para la comprensión. Guía para el docente. Buenos Aires. Paidós.

Cañizalez Mesa, N. E., \& Benavides Rozo, F. A. (2018). La didáctica como herramienta de la reflexión docente. Educación Y Territorio, 6(11), 105 - 145. Recuperado a partir de https://jdc.edu.co/revistas/index.php/reyte/article/ view $/ 48$

Castañeda, R. A., \& Tabares, A. M. (2015). Secuencia didáctica de enfoque interactivo para comprender textos narrativos en tercero de primaria. (Tesis de maestría). Universidad Tecnológica de Pereira, Risaralda, Colombia. Recuperado de www.google.com.co/webhp?sourceid=chrome

Colbert, V., \& VÁsquez, L. N. (2009). Escuela Nueva - Escuela Activa. Manual para el docente. Bogotá, Colombia: Fundación Escuela Nueva Volvamos a la Gente.

Coronel, D. E. (2015). Implementar estrategias didácticas en el uso del juego lúdico para mejorar la enseñanza de la lectura, para que los estudiantes incrementen acciones como relacionar y decodificar. (Tesis de maestría). Universidad de Carabobo, Estado de Carabobo, Venezuela.

Duarte, J. (2003). Ambientes de aprendizaje una aproximación conceptual. Estudios Pedagógicos, (29), 97-113. Recuperado de https://scielo.conicyt.cl/ scielo.php?pid=S0718-07052003000100007\&script=sci_arttext\&tlng=pt

ElLiotT, J. (2002). Investigación Acción en Educación. Madrid: Editorial Morata, S. L.

Henao, O. (2011). Comprensión lectora de los alumnos de educación básica primaria: una evaluación en Escuelas públicas de Medellín. Revista Interamericana de Bibliotecología, 1(24), 45-67.

ICFES, (2012). Cartilla Preguntas analizadas, Lenguaje $3^{\circ}$. Recuperado de http://www.icfes.gov.co/docman/estudiantes-y-padres-de-familia/ejemplosde-preguntas2/ejemplos-de-preguntas-saber-3-5-y-9/grado-3/876-ejemplosde-preguntas-saber-3-lenguaje 
Jolibert, J. (2002). Formar niños lectores de textos. España: Dolmen ediciones S.A. Mogollón, O., \& Solano, M. (2011). Escuelas activas. Apuestas para mejorar la calidad de la educación. Recuperado de https://www.google.com.co/searc $\mathrm{h} ? \mathrm{q}=$ escuelas + activas + apuestas + para + mejorar $+\mathrm{la}+$ calidad + de + la + educaci on\&

Samacá Bohórquez, I. (2016). El espíritu científico en la primera infancia. Praxis \& Saber, 7(13), 89 - 106. https://doi.org/10.19053/22160159.4167

Orrego Noreña, J., \& Toro GonzÁlez, L. (2014). Relaciones vitales: el aula como escenario permanente de investigación. Praxis E Saber, 5(10), 121 - 139. https://doi.org/10.19053/22160159.3025

Sindicato de Maestros de Boyacá (1994). Ley general de Educación, Ley 115 de 1994.

SolÉ, I. (2011). Estrategias de Lectura. Barcelona, España: Editorial Graó.

SolÉ, I. (1992). Estrategias de Lectura. Barcelona, España: Editorial Graó.

Thorne, C., Morla, K., Nakano, T., Mauchi, B., Landeo, L., Huerta, R., \& Angie, V. (2012). Estrategias de comprensión de lectura mediadas por TIC. Una alternativa para mejorar las capacidades lectoras en primaria. Recuperado de https://dash.harvard.edu/bitstream/handle/1/11654978/Thorne_Morla_ Uccelli_et_al_2013.pdf?sequence.pdf 